\title{
Cutoff due to pointwise degradations in color images
}

\author{
Alex Golts* and Yoav Y. Schechner \\ Department of Electrical Engineering, Technion-Israel Institute of Technology, Haifa 32000, Israel \\ *Corresponding author: alexgo@tx.technion.ac.il
}

Received May 19, 2014; revised September 25, 2014; accepted October 1, 2014;

posted October 3, 2014 (Doc. ID 210086); published November 14, 2014

\begin{abstract}
Many studies analyze resolution limits in single-channel, pan-chromatic systems. However, color imaging is popular. Thus, there is a need for its modeling in terms of resolving capacity under noise. This work analyzes the probability of resolving details as a function of spatial frequency in color imaging. The analysis introduces theoretical bounds for performance, using optimal linear filtering and fusion operations. The work focuses on resolution loss caused strictly by noise, without the presence of imaging blur. It applies to full-field color systems, which do not compromise resolution by spatial multiplexing. The framework allows us to assess and optimize the ability of an imaging system to distinguish an object of given size and color under image noise. (C) 2014 Optical Society of America

OCIS codes: (350.5730) Resolution; (010.1690) Color; (110.0110) Imaging systems; (110.4280) Noise in

imaging systems; (040.3780) Low light level; (110.3000) Image quality assessment.

http://dx.doi.org/10.1364/JOSAA.31.002711
\end{abstract}

\section{INTRODUCTION}

Many imaging problems are characterized by pointwise image formation models $[\underline{1}, 2]$. These models treat each pixel independent of its surroundings, unlike effects such as optical blur. Such problems include specularities over a diffuse reflection [3,4], flash photography [5] , attenuation and path radiance in haze [ $\underline{6}-11]$, and dirt on windows [12]. Even in such pointwise problems, which lack optical blur, noise degrades the effective resolution (see Fig. 1), as shown in [2]. However, the analysis in [2] applies to single-channel, monochrome sensors.

Color imaging is common. Medicine [13], astronomy [14], and defense [15] are just a few domains of use. Color imaging requires the capture of several spectral measurements. Some systems use more than the red-green-blue (RGB) trichromatic channels. A statistical study of hyperspectral images is presented in [16]. For color, some psycho-physical studies were conducted, in an effort to gain better understanding of human vision [17-20]. Practical approaches for resolution enhancement using data from multiple image sensors have also been presented [21-23].

Several image acquisition techniques exist for color imaging. Some systems split the light and project it onto (typically three) separate monochrome sensors, using a prism. Different forms of time-multiplexing techniques are also available, typically for hyperspectral imaging [24-29]. In this concept, one sensor is used for capturing all spectral channels, at different times. Another possibility is to use spatial-multiplexing techniques. Here, the spectral channels are captured across different areas of a single monochrome sensor. As a result, spatial resolution is compromised. Spatial-multiplexing was demonstrated using a prism [30]. However, the most popular way of generating color images is using a color filter array (CFA). A generalized CFA approach has been proposed for hyperspectral imaging [31]. For RGB images, a Bayer pattern CFA [32] is commonly used. Reference [33] discusses Bayer sensors and their inherent resolution limitations.
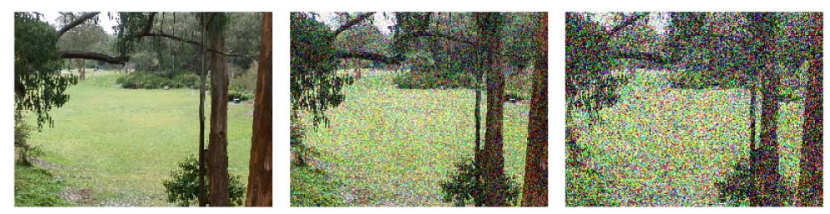

Fig. 1. From left to right: original image, image corrupted by white Gaussian noise, image corrupted by twice stronger white Gaussian noise. Fine details, such as small branches and grass are lost, as the level of noise increases.

Noise and resolution analysis are part of performance prediction models. These models are required as a tool for system engineers during design. Sometimes, such models are also used when the possibility to perform actual experiments is limited due to high risk or cost [34]. Most available performance prediction models are for single-channel systems, such as pan-chromatic visible light sensors or single-channel infrared sensors [35].

Our work focuses on full-field color imaging systems, which, unlike Bayer, do not fundamentally degrade resolution. We show that a system's spatial resolution can be enhanced by multispectral information, in comparison to a pan-chromatic imaging system.

This paper derives limiting cutoff frequency under noise, in color imaging. The analysis is irrespective of a potential human in the process. This paper generalizes a prior study on limits in monochrome images [2] . The analysis in our study accounts for inherent detector noise, as well as potential noise filtering, which is optimally derived for each color channel. It also considers optimal image fusion, which can be viewed as filtering as well, albeit in the color domain. The goal is to assess, within a desired success rate, the spatial cutoff frequency, given color specifications of a system and scenario. An imaging system's effective cutoff also defines its smallest recoverable target size. 


\section{BACKGROUND}

\section{A. Pointwise Degradations}

Let $l_{c}^{\text {object }}(\mathbf{x})$ be the image irradiance of an object acquired at pixel $\mathbf{x}=(x, y)$, in color channel $c$, under ideal conditions, without any degradation effects. Due to pointwise degradation effects, the measured signal in each color channel takes the form

$$
I_{c}(\mathbf{x})=l_{c}^{\text {object }}(\mathbf{x}) t_{c}(\mathbf{x})+a_{c}(\mathbf{x})+n_{c}(\mathbf{x}) .
$$

Here, $t_{c}(\mathbf{x})$ and $a_{c}(\mathbf{x}) \geq 0$ are, respectively, deterministic multiplicative and additive effects per color channel [36]. The pointwise model applies to a wide range of problems. For example, $t_{c}(\mathbf{x})$ can be a distance-dependent atmospheric transmittance [1,2]. Then, $a_{c}(\mathbf{x})$ accounts for path radiance (airlight) [10]. In addition, $n_{c}(\mathbf{x})$ accounts for random noise. The $c$ dependency generalizes the monochrome model given in [2]. We aim to assess resolution limits in color imaging systems. We account for loss of details due to pointwise degradations, such as random noise. Usually, pointwise degradations are considered as effects which reduce sensitivity, rather than resolution.

According to [1, 2, $37-39]$, the overall noise variance (assuming a camera without amplifier nonlinearities $[40,41])$ in the image is $\sigma_{c}^{2}(\mathbf{x})=\chi_{c} \tilde{I}_{c}(\mathbf{x})+\psi_{c}$, where $\chi_{c}, \psi_{c}>0$, while $\tilde{I}_{c}(\mathbf{x})$ is the expected image intensity given in Eq. (1), excluding $n_{c}(\mathbf{x})$. The term $\chi_{c}$ parameterizes signal-dependent photon (shot) noise [42,43]. The term $\psi_{c}$ expresses signalindependent random noise components. As detailed in $[39, \underline{42}, \underline{44}], \psi_{c}=\rho_{c \text {-read }}^{2}+\rho_{c \text {-digit }}^{2}+D_{c} T_{c}$, where $\rho_{c \text {-read }}$ is the detector readout noise standard deviation (STD), $\rho_{c \text {-digit }}$ is the noise STD of the sensor quantizer, $D_{c}$ is the detector dark current, and $T_{c}$ is the exposure time.

Following Eq. (1), the signal-to-noise (SNR) can be different between color channels. One reason is that noise variance depends on the local image signal. The non-negative additive component $a_{c}(\mathbf{x})$ also has spectral dependency. In Section 3 we account for variability in SNR among the color channels, suggesting an optimal fusion process in the color domain, that suits a given color distribution of SNR values.

The model presented here for pointwise degradations is meant to describe a category of problems that our model for limiting cutoff frequency applies to. Some works focus on estimating various model parameters such as $t_{c}(\mathbf{x})$ and $a_{c}(\mathbf{x})$, in various scenarios. In this work we study the fundamental resolution limits due to unknown random noise and not due to a systematic error of model parameters. If there is such a systematic error, it of course biases the error further. We do not aim to estimate these parameters in this work. They are assumed to be known.

\section{B. Monochrome Resolution Cutoff}

The ability to recover an object of given size depends on the SNR [2]. At low spatial frequencies, the signal can be distinguished even for very low SNR, contrary to the situation in higher spatial frequencies. Reference [2] finds a monochrome effective cutoff frequency due to random noise, within a desired success rate of object recovery. The analysis also accounts for potential linear noise filtering. The notion of frequency response and thus the limiting cutoff frequency is enabled by linear systems theory. Given an effective limiting cutoff frequency $u_{\text {cutoff }}[\mathrm{cy} /$ pixel], the transversal length of the smallest object that can be recovered successfully is [2]

$$
m=\frac{1}{2 u_{\text {cutoff }}} \text { [pixels]. }
$$

Consider a single-channel image signal of the form

$$
s(\mathbf{x})=A \cos \left(2 \pi u^{\prime} x\right) .
$$

It has horizontal spatial frequency $u^{\prime} \in\left[0, \frac{1}{2}\right]$ cy/pixel and amplitude $A$. This signal is corrupted by white Gaussian noise with STD $\sigma$. A signal at a specific spatial frequency is studied, since it indicates the potential of recovering an object of given size [Eq. (2)]. Similar to Ref. [2], we define the SNR of such an input signal as

$$
\mathrm{SNR}_{\text {in }}=\frac{|A|}{\sigma} .
$$

Reference [2] shows that applying a linear averaging window filter can increase an output SNR. The maximum increase is achieved for a window of size

$$
W_{\max }(u)=(2 u)^{-1}
$$

Note that the maximal window size is constrained by the signal spatial frequency. If a larger window size was chosen, signal details would be lost.

The SNR increase factor for this optimal window size is

$$
C_{\max }(u)=\frac{1}{\sin (\pi u)}
$$

The SNR in the processed image is thus

$$
\operatorname{SNR}_{\text {out }}(u)=C_{\text {max }}(u) \operatorname{SNR}_{\text {in }}(u) .
$$

Let $\rho \in[0,1]$ be a minimum required rate of successful object recovery. The rate $\rho$ imposes [2] a minimal required output SNR

$$
\mathrm{SNR}_{\text {out }}^{\min }=\operatorname{erf}^{-1}(\rho)
$$

Substituting Eq. (6) into Eq. (7), the maximum resolvable cutoff spatial frequency is

$$
u_{\text {cutoff }}=\frac{1}{\pi} \cdot \arcsin \left(\frac{\mathrm{SNR}_{\mathrm{in}}}{\mathrm{SNR}_{\mathrm{out}}^{\min }}\right) .
$$

Spatial filtering using $W_{\max }(u)$ is not meant [2] to be a practical denoising method. It rather serves as an accessory to obtaining a closed-form expression for a resolution bound, $u_{\text {cutoff }}$. In Section 3, this approach for assessing the limiting cutoff is extended to color images.

\section{FULL-FIELD COLOR IMAGES}

We extend the principles of Section 2.B to color imaging systems. We focus on general systems, putting aside human perception considerations. In this work, we discuss high-end imaging systems that, unlike Bayer-based sensors [33], do not compromise resolution by spatial multiplexing. A prism-based 
3CCD system is the most common example. Other designs include N-CCD $(N>3)$, time multiplexing (e.g., a camera with an external spectral filter wheel), and FOVEON X3 technology $[45,46]$. They consist of three distinct sensor arrays corresponding to the R, G, and B channels. The Bayer sensor, a popular means of acquiring color images, is outside the scope of this paper. Its inherent resolution loss is analyzed separately in Ref. [33].

Assume $N$ image channels. Each channel $c$ contains a signal as in Eq. (3)

$$
s_{c}(\mathbf{x})=A_{c} \cos \left(2 \pi u^{\prime} x\right)+B_{c},
$$

where $A_{c}$ is the per-color signal amplitude, and $B_{c}$ is a constant ("DC") background level. The values of $\left\{A_{c}\right\}$ and $\left\{B_{c}\right\}$ jointly define the color of the object. A per-color phase component is neglected following analysis in [33]. The noise STD in each channel is $\sigma_{c}$. The SNR per channel is

$$
\operatorname{SNR}_{c}^{\text {in }}(u)=\frac{\left|A_{c}\right|}{\sigma_{c}}
$$

An example of such a signal is shown in Fig. 2, in RGB representation. It is a yellow signal over a green background. We can see that the variations are easier to distinguish in low spatial frequencies, or high SNR. Figure 2 is a sample color representation of a monochrome plot [2] . The solid black line represents a monochrome cutoff, according to [2]. The dotted horizontal arrow denotes $\mathrm{SNR}_{\text {out }}$. Below this arrow, the signal's $\mathrm{SNR}$ is greater than $\mathrm{SNR}_{\text {out }}^{\min }$, and the formula for the limiting cutoff frequency [Eq. (9)] is not used. When the SNR is greater than this threshold, there is no need for averaging filtering as described in Section 2.B. A signal's probability of recovery is independent of spatial frequency when its raw $\mathrm{SNR}$ is above the minimal threshold $\mathrm{SNR}_{\text {out }}^{\min }$.

\section{A. Filtering in the Spectral/Color Domain}

A technique for SNR optimization [47] is linear fusion based on the Rayleigh principle [48]. It ultimately results in a weighted sum of the color channels: a linear filter in the color domain. A measured signal level is

$$
\hat{s}_{c}(x, y)=s_{c}(x, y)+n_{c}(x, y)
$$

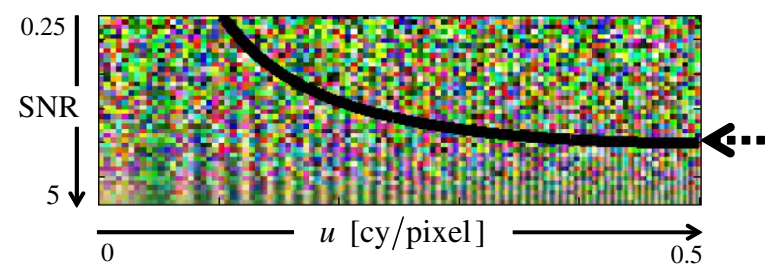

Fig. 2. RGB signal of the form $B_{c}+A_{c} \sin \left(2 \pi u^{\prime} x\right)$, where $B_{c}$ is a bias, $A_{c}$ is the signal amplitude, $u^{\prime} \propto x$, and $c \in[R, G, B]$. Spatially uncorrelated (white) Gaussian noise is added to each channel. Its variance increases along the vertical axis. Beyond the solid black line, in the upper-right corner, it becomes very difficult to distinguish the signal from the background. This line represents the limiting cutoff frequency for a $50 \%$ success chance $(\rho=0.5)$, without accounting for the potential benefit of color information (Section 2.B). The dotted horizontal arrow corresponds to $\mathrm{SNR}_{\mathrm{out}}^{\mathrm{min}}$. Below this arrow, all spatial frequencies are resolvable. A signal with any spatial frequency can be recovered if its SNR is large enough. In our work we show that by utilizing color information, the limiting cutoff denoted by the solid line is increased. where $s_{c}(x, y)$ is given by Eq. $(\underline{10})$, and $n_{c}(x, y) \propto \mathcal{N}\left(0, \sigma_{c}^{2}\right)$ is spatially uncorrelated (white) Gaussian noise with STD $\sigma_{c}$. The vector of measured values is $\tilde{\mathbf{s}}(x, y)=\left[\tilde{s}_{1}(x, y)\right.$, $\left.\tilde{s}_{2}(x, y), \ldots, \tilde{s}_{N}(x, y)\right]^{T}$, where $T$ denotes transposition. Using vector notations for the signal and noise in all image channels

$$
\tilde{\mathbf{s}}(x, y)=\mathbf{s}(x, y)+\mathbf{n}(x, y) .
$$

A fused image is a weighted sum of the raw multichannel pixel data

$$
g(x, y)=\langle\boldsymbol{\alpha}, \tilde{\mathbf{s}}(x, y)\rangle=\sum_{c=1}^{N} \alpha_{c} \tilde{s}_{c}(x, y),
$$

where \langle\rangle is the inner product operator, $\alpha$ is a pixelwise weight vector, and $\alpha_{c}$ is its $c$ th element.

The respective powers of the signal and noise in the fused image are

$$
\left\|g_{\mathbf{A}}(x, y)\right\|^{2}=\boldsymbol{\alpha}^{T} \mathbf{A}(x, y) \mathbf{A}^{T}(x, y) \boldsymbol{\alpha},
$$

$$
\left\|g_{\mathbf{n}}(x, y)\right\|^{2}=\boldsymbol{\alpha}^{T} \mathbf{n}(x, y) \mathbf{n}^{T}(x, y) \boldsymbol{\alpha} .
$$

Let

$$
\mathbf{C}_{\mathbf{A}}=\mathbf{A}(x, y) \mathbf{A}^{T}(x, y)
$$

be the cross-color signal amplitude correlation matrix, and

$$
\mathbf{C}_{\mathbf{n}}=E\left\{\mathbf{n}(x, y) \mathbf{n}^{T}(x, y)\right\}
$$

be the noise covariance matrix, where $E$ is the expectation operator. Since $n_{c}(x, y) \propto \mathcal{N}\left(0, \sigma_{c}^{2}\right), \mathbf{C}_{\mathbf{n}}=\operatorname{diag}\left[\sigma_{c}^{2}\right]$.

The square of the local SNR in the fused image is

$$
\operatorname{SNR}^{2}(x, y)=\frac{E\left\{\left\|g_{\mathbf{A}}(x, y)\right\|^{2}\right\}}{E\left\{\left\|g_{\mathbf{n}}(x, y)\right\|^{2}\right\}}=\frac{\boldsymbol{\alpha}^{T} \mathbf{C}_{\mathbf{A}} \boldsymbol{\alpha}}{\boldsymbol{\alpha}^{T} \mathbf{C}_{\mathbf{n}} \boldsymbol{\alpha}} .
$$

Equation (19) is a generalization of Rayleigh's quotient [48]. We seek a weight vector $\boldsymbol{\alpha}$ that maximizes the SNR. Then, the gradient of Eq. (19) with respect to $\boldsymbol{\alpha}$ is null. Since $\mathbf{C}_{\mathbf{n}}$ is positive-definite, this requirement leads to an eigenvalue problem

$$
\mathbf{C}_{\mathbf{n}}^{-1} \mathbf{C}_{\mathbf{A}} \boldsymbol{\alpha}=\mathrm{SNR}^{2} \boldsymbol{\alpha}
$$

Note that $\mathrm{SNR}^{2}$ is the eigenvalue of $\mathbf{C}_{\mathbf{n}}^{-1} \mathbf{C}_{\mathbf{A}}$, and $\boldsymbol{\alpha}$ is the corresponding eigenvector.

By the Rayleigh principle [48], the SNR of the fused image is maximized if $\boldsymbol{\alpha}$ is the eigenvector corresponding to the largest eigenvalue $\mathrm{SNR}_{\max }^{2}$ of $\mathbf{C}_{\mathbf{n}}^{-1} \mathbf{C}_{\mathbf{A}}$. The vector $\boldsymbol{\alpha}$ is normalized so that $\sum_{c=1}^{N} \alpha_{c}=1$. The maximum SNR following spectral fusion is $\mathrm{SNR}_{\max }$. It is the square root of the largest eigenvalue.

Equation (14) yields a monochrome image. This image is then analyzed as in [2], using an optimal averaging window per spatial frequency [Eq. (5)]. This completes a spatiospectral enhancement process.

Next, we demonstrate the benefit due to the proposed fusion process. For this purpose, we define a gain factor. It is the pixelwise ratio between the SNR of an optimally fused image 
(a)

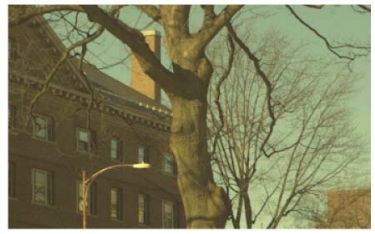

(b)

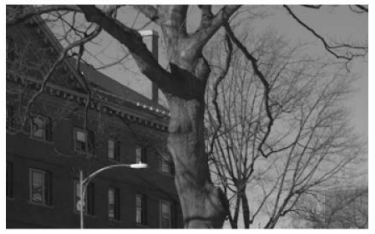

Fig. 3. (a) Original hyperspectral image [16] rendered in RGB. (b) Original image, as it would appear in a pan-chromatic camera.

and the SNR of a pan-chromatic image. The latter is equivalent to the choice of a spectrally uniform weight vector $\boldsymbol{\alpha}$. The gain factor is

$$
D_{\text {max }} \equiv \frac{\mathrm{SNR}_{\text {fused }}^{\text {out }}}{\mathrm{SNR}_{\text {pan }}^{\text {in }}},
$$

where $\mathrm{SNR}_{\text {pan }}^{\text {in }}$ is the pan-chromatic $\mathrm{SNR}$, and $\mathrm{SNR}_{\text {fused }}^{\text {out }}$ is the $\mathrm{SNR}$ following optimal color fusion (equivalent to $\mathrm{SNR}_{\max }$ ).

\section{B. Demonstration}

We applied our color fusion approach to a hyperspectral image in the visible spectral range. The image was taken from the database used in [16]. It consists of $1392 \times 1040$ pixels and 31 spectral channels. Figure 3(a) shows the image we chose, rendered in RGB. Figure $3(\mathrm{~b})$ shows how it would appear, had it been captured with a pan-chromatic camera: photons from all 31 channels are simply integrated. The images used in [16] were captured in good lighting conditions, using a quality camera with high spatial resolution. Therefore, noise is not significant in Fig. 3. The benefit of our approach is most dominant when pointwise degradations such as noise are apparent. So, we simulate image capture in two types of noisy conditions. The original noiseless image in spectral channel $c$ is $s_{c}$. We then add random noise with STD $\sigma_{c}$ to each pixel in every image channel. The resulting noisy image is $\hat{s}_{c}$.

In order to fuse the hyperspectral channels according to Section 3.A, we need pixelwise estimates of $A_{c}$. In practice, (a)

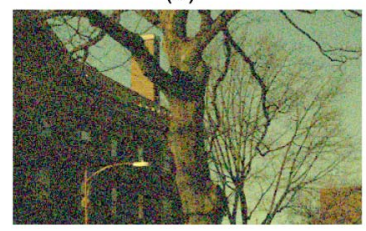

(c)

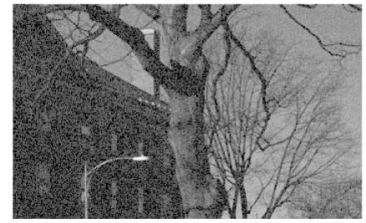

(b)

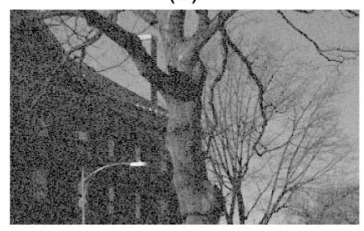

(d)

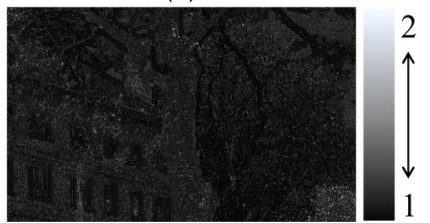

Fig. 4. (a) RGB-rendered image for low variability $\mathrm{SNR}_{c}^{\text {in }}$. (b) Same image, as it would appear in a pan-chromatic camera. (c) Optimally fused image, for low variability $\mathrm{SNR}_{c}^{\text {in }}$. Only slight improvement may be noticed, e.g., in distinguishing the left side of the chimney from the sky background. (d) Pixelwise ratio between the SNR of the optimally fused image to the SNR of the pan-chromatic image, $D_{\max }(x, y)$. It never goes below 1 ; thus the fused image is never degraded compared to the pan-chromatic case. Here, the gain goes up to almost 2, but mostly it takes values closer to 1 . natural images contain many spatial frequency components. However, we may estimate the signal amplitude locally by a localized contrast estimation of $s_{c}$, the noiseless image

$$
\left|A_{c}(x, y)\right|=\left|s_{c} *\left(1-h_{\text {avg }}\right)\right|,
$$

where $h_{\text {avg }}$ is a $5 \times 5$ averaging filter. This yields the differential signal of each pixel relative to an estimate of its close background level. This signal estimation is used along with the noise STD $\sigma_{c}$ in the pixelwise calculation of the optimal weight vector $\boldsymbol{\alpha}(x, y)$.

Note again that we do not propose a denoising or spectral fusion method. This linear process is done to demonstrate our approach for assessing resolution capabilities in color images, corrupted by noise.

The noise is estimated at each pixel location according to the noise model from Section 2.A, $\sigma_{c}^{2}(\mathbf{x})=\chi_{c} s_{c}(\mathbf{x})+\psi_{c}$. We used $\chi_{c}=1$, while $\psi_{c}$ was chosen differently in each of the two scenarios that we analyzed.

\section{Low Variability $\mathrm{SNR}_{c}^{\mathrm{in}}$}

In this scenario, we add noise with an identical $\psi_{c} \forall c$. This component was chosen to be dominant relative to the signaldependent photon noise. In haze or fog, intense path radiance reaches the camera in all spectral channels. The scattering is dominant compared to the signal, so the photon noise has a low variability across $c$, and so does the SNR. Low variability SNR also occurs in cameras limited by spectrally constant readout noise. Figure 4(a) shows the image obtained in this case, rendered in RGB. Figure 4(b) shows the same image, as it would appear in a pan-chromatic camera. Figure $\underline{4(c)}$ shows the resulting fused image, after performing color fusion as explained in Section 3.A. In this scenario the two images are very similar, with only slight improvement due to fusion. Figure $4(\mathrm{~d})$ shows $D_{\max }(x, y)$, the optimal SNR gain due to color fusion [Eq. (21)].

\section{High Variability $\mathrm{SNR}_{c}^{\mathrm{in}}$}

Now, we add noise with the component $\psi_{c}$ inversely proportional to the camera's spectral sensitivity. The reason is that channels with low sensitivity may require longer exposure time, thus increasing dark current. Another possible reason is the selection of a larger amplifier in channels with low (a)

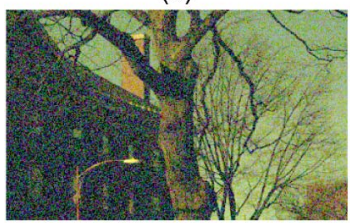

(c)

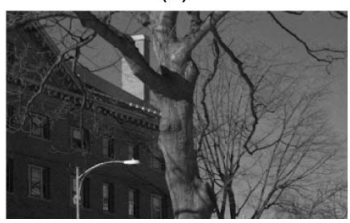

(b)

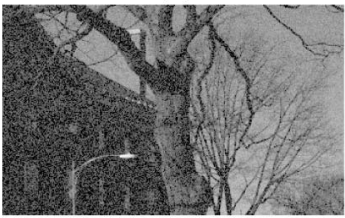

(d)

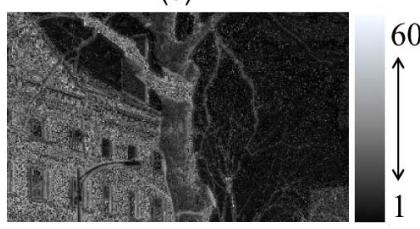

Fig. 5. (a) RGB-rendered image for high variability $\mathrm{SNR}_{c}^{\text {in }}$. (b) Same image, as it would appear in a pan-chromatic camera. (c) Optimally fused image, for high variability $\mathrm{SNR}_{c}^{\mathrm{in}}$. Significant improvement is noticed in distinguishing small objects across the image. (d) The pixelwise ratio, $D_{\max }(x, y)$, between the SNR of the optimally fused image and the SNR of the pan-chromatic image. Here, gain is up to nearly 62 . 
sensitivity, thus increasing their readout noise. Figure $\underline{5(a)}$ shows the image obtained in this case, rendered in RGB. Figure 5(b) shows the same image, as it would appear in a panchromatic camera. Figure 5(c) shows the resulting fused image, after color fusion. Major improvement is obtained in distinguishing details compared to the image in Fig. 5(b). Figure $\underline{5}$ (d) shows $D_{\max }(x, y)$ for this scenario.

\section{CUTOFF IN FULL-FIELD SYSTEMS}

We compare the optimal SNR resulting from the suggested color fusion process to the SNR obtained in a pan-chromatic image. The pan-chromatic case implies a uniform weight $\boldsymbol{\alpha} \approx[0.333,0.333,0.333]$. It is important to note that the total number of photons is set to be equal in both color and the pan-chromatic images. This provides a "fair" comparison. It ensures that any observed improvement in $u_{\text {cutoff }}$ is due to optimal utilization of multispectral data, even though the total number of photons is equal in both cases. The SNR per color channel, however, may vary (see Section 2.A).

The SNR following optimal color fusion (See Appendix A) is

$$
\mathrm{SNR}_{\text {fused }}^{\text {out }}=\sqrt{\sum_{c=1}^{N}\left[\mathrm{SNR}_{c}^{\mathrm{in}}\right]^{2}}
$$

where $\left\{\mathrm{SNR}_{c}^{\mathrm{in}}\right\}$ are the input SNR values of the $N$ color channels. The optimal SNR gain $D_{\max }$ then becomes

$$
D_{\text {max }} \equiv \frac{\mathrm{SNR}_{\text {fused }}^{\text {out }}}{\mathrm{SNR}_{\text {pan }}^{\text {in }}}=\frac{1}{\mathrm{SNR}_{\text {pan }}^{\text {in }}} \cdot \sqrt{\sum_{c=1}^{N}\left[\mathrm{SNR}_{c}^{\text {in }}\right]^{2}} .
$$

The effect of polychromaticity on the maximum resolvable cutoff frequency, $u_{\text {cutoff }}$, is seen by generalizing Eq. (7) to

$$
\operatorname{SNR}_{\text {out }}(u)=C_{\text {max }}(u) D_{\text {max }} \mathrm{SNR}_{\text {pan }}^{\text {in }}
$$

Consequently, the expression for $u_{\text {cutoff }}$ in Eq. (9) becomes

$$
u_{\text {cutoff }}=\frac{1}{\pi} \cdot \arcsin \left(\frac{D_{\mathrm{max}} \mathrm{SNR}_{\mathrm{pan}}^{\mathrm{in}}}{\mathrm{SNR}_{\mathrm{out}}^{\mathrm{min}}}\right) .
$$

Figure 6 shows the cutoff frequency, $u_{\text {cutoff }}$ for two different success rates $\rho$ [related to $\mathrm{SNR}_{\text {out }}^{\min }$ through Eq. (8)], as a function of $D_{\text {max }} \mathrm{SNR}_{\text {pan }}^{\text {in }}$. Unlike pan-chromatic images, low input

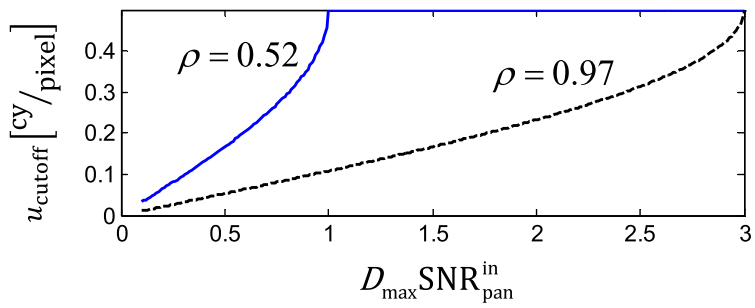

Fig. 6. Limiting cutoff frequency, $u_{\text {cutoff }}$, as a function of $D_{\max } \cdot \mathrm{SNR}_{\mathrm{pan}}^{\mathrm{in}}$, for two different success rates, $\rho$. The blue solid line corresponds to $\mathrm{SNR}_{\text {out }}^{\min }=1$, which accounts for a success rate of $\rho=$ 0.52 [Eq. (8)]. The black dotted line corresponds to $\mathrm{SNR}_{\text {out }}^{\min }=3$, which accounts for a success rate of $\rho=0.97$.
$\mathrm{SNR}_{\text {pan }}^{\text {in }}$ can be amplified by a high value of $D_{\max }$, yielding a high cutoff frequency.

\section{A. Examples}

1. Numerical Examples

Let us consider two scenarios. In one, the SNR differs moderately across color channels. The other consists of an object with a very specific color (red) signal over an arbitrarily colored background. We consider both scenarios to be dominated by photon noise, due to the background "DC" signal. In the first scenario, consider an image whose RGB signal amplitude and background (given in photoelectrons) are, respectively,

$$
\begin{aligned}
& \left\{A_{c}\right\}=[120,100,90] \\
& \left\{B_{c}\right\}=[10000,15000,5000] .
\end{aligned}
$$

The photon noise STD vector is

$$
\sigma_{c} \approx[\sqrt{10000}, \sqrt{15000}, \sqrt{5000}]
$$

The resulting input $\mathrm{SNR}$ vector is $\left\{\mathrm{SNR}_{c}^{\mathrm{in}}\right\}=[1.2,0.82,1.27]$. The color fusion process from Section 3.A yields an optimal output SNR

$$
\mathrm{SNR}_{\text {fused }}^{\text {out }}=1.93 \text {, }
$$

using the weight vector $\boldsymbol{\alpha}=[0.33,0.18,0.49]$. The panchromatic SNR is obtained by a uniform $\alpha$. Then,

$$
\mathrm{SNR}_{\mathrm{pan}}^{\mathrm{in}}=\frac{\sum_{c=1}^{3} A_{c}}{\sqrt{\sum_{c=1}^{3} \sigma_{c}^{2}}}=1.79
$$

Thus, from Eqs. (29) and (30)

$$
D_{\max } \approx 1.08 \text {. }
$$

For $\mathrm{SNR}_{\text {out }}^{\mathrm{min}}=3$, which corresponds to a $\rho=0.97$ success rate, we obtain a cutoff frequency [Eq. (26)]

$$
u_{\text {cutoff }}^{\text {fused }}=0.22\left[\frac{\text { cy }}{\text { pixel }}\right] \text {. }
$$

In the pan-chromatic case, we use Eq. (9), or apply $D_{\max }=1$ in Eq. (26) to obtain

$$
u_{\text {cutoff }}^{\text {pan }}=0.20\left[\frac{\text { cy }}{\text { pixel }}\right] \text {. }
$$

Here, $u_{\text {cutoff }}^{\text {pan }}$ is $9 \%$ less than $u_{\text {cutoff }}^{\text {fused }}$.

In the second scenario, consider the following signal (in electrons):

$$
\begin{aligned}
& \left\{A_{c}\right\}=[300,0,0], \\
& \left\{B_{c}\right\}=[10000,15000,5000] .
\end{aligned}
$$

It is a red object over a yellowish bright background. The photon noise STD vector can be assumed the same as in Eq. (28), since background signal is dominant. Thus, $\mathrm{SNR}_{\text {in }}=$ $[3,0, \overline{0}]$. The color fusion process from Section 3.A locks on the $\mathrm{R}$ channel alone. This yields an optimal output SNR 


$$
\mathrm{SNR}_{\text {fused }}^{\text {out }}=3 \text {, }
$$

using the weight vector $\boldsymbol{\alpha}=[1,0,0]$. The pan-chromatic SNR obtained by Eq. (30) is $\mathrm{SNR}_{\text {pan }}^{\text {in }}=1.73$, thus $D_{\max }=1.73$. Taking $\mathrm{SNR}_{\text {out }}^{\min }=3$, which corresponds to a $\rho=0.97$ success rate, we obtain a cutoff frequency

$$
u_{\text {cutoff }}^{\text {fused }}=0.5\left[\frac{\text { cy }}{\text { pixel }}\right] \text {. }
$$

In the pan-chromatic case

$$
u_{\text {cutoff }}^{\text {pan }}=0.20\left[\frac{\mathrm{cy}}{\text { pixel }}\right]
$$

Here, $u_{\text {cutoff }}^{\text {pan }}$ is $60 \%$ less than $u_{\text {cutoff }}^{\text {fused }}$.

We see that depending on the scenario, the benefit of polychromatic imaging can vary a lot. If the variability in SNR across the color channels is small, then it contributes moderately. If the variability is large, it can enable discrimination of much smaller objects.

\section{Simulation-Varying Spatial Frequency}

Figure 7 emphasizes the improvement that can be achieved using three image channels versus pan-chromatic imaging. Here, the pan-chromatic SNR, SNR pan, varies between 0.25 and 5 in the vertical axis. The noise variance is identical in all three channels. The signal was chosen as

$$
\left\{A_{c}\right\}=[0,15,0],
$$

while $u^{\prime} \propto x$

Figure 7(a) shows the signal, rendered in RGB. The solid blue line represents the limiting cutoff frequency for a $50 \%$ success chance $(\rho=0.5)$, without accounting for the potential benefit of color information (see Section 2.B).

Figure 7(b) shows the same signal, as it would appear in a pan-chromatic camera $(\boldsymbol{\alpha} \approx[0.333,0.333,0.333])$. Figure $7(\mathrm{c})$ shows the result of the color fusion process. In Fig. $\underline{7(\mathrm{c})}$, the SNR is increased relative to Fig. $7(\mathrm{~b})\left(\mathrm{SNR}_{\text {pan }}^{\mathrm{in}}\right)$ by a $D_{\max }=$ $\sqrt{3}$. Note that the solid blue line in Fig. $7(\mathrm{c})$ is closer to the upper-right corner, meaning that the limiting cutoff frequency has increased, for the same input SNR. This improvement is obtained due to the optimal utilization of multispectral information. In this example, the signal is fully associated with the green channel, thus the chosen weight vector is $\alpha \approx[0,1,0]$.

\section{B. What If the Signal Is Uncertain?}

Similar to the spatial filtering in the monochrome case (see Section 2.B), note that the spectral fusion process presented in Section 3.A is not meant for image processing. We rather use it as a step in obtaining the expression for the limiting cutoff frequency, $u_{\text {cutoff }}$. If this method was to be used for fusion in practice, an estimation of $\mathbf{A}(x, y)$ and $\mathbf{n}(x, y)$ would be required.

Here, we show an example for the effect of uncertainty in the per-channel signal components, $\left\{A_{c}\right\}$. Suppose the noise STD is known. Inaccurate $\left\{A_{c}\right\}$ implies suboptimal fusion weight vector $\boldsymbol{\alpha}$, and thus suboptimal SNR [Eq. (19)]. This yields an underestimated cutoff frequency [Eq. (26)]. As an example, we take the per-channel signal and noise distribution of the first scenario presented in Section 4.A.1. Figure 8 (a)

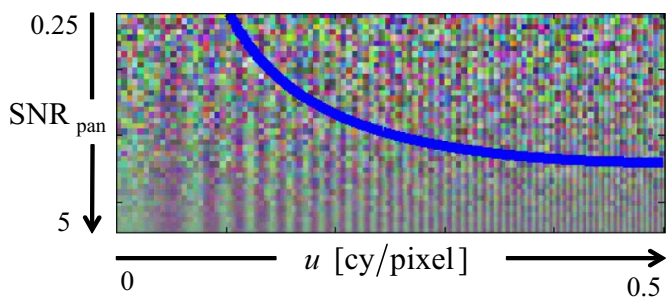

(b)

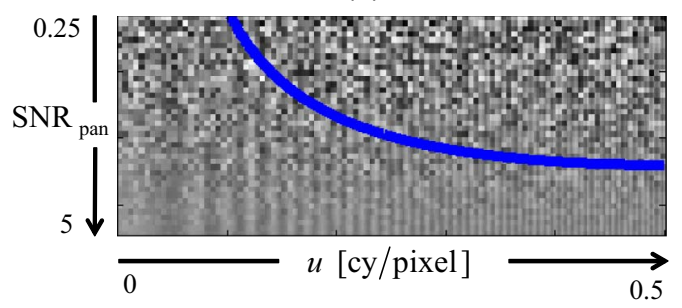

(c)

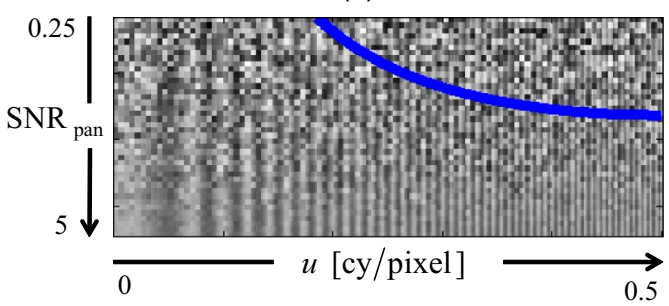

Fig. 7. (a) RGB-rendered signal with varying SNR and spatial frequency. (b) The same signal, as it would be seen in a pan-chromatic camera. (c) The signal after optimal fusion in the color domain. The cutoff frequency, represented by the solid blue line, has increased.

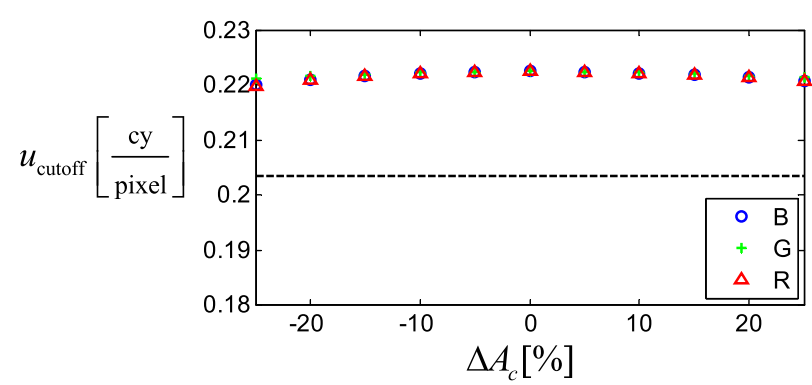

Fig. 8. Limiting cutoff frequency as a function of the relative estimation error of each $A_{c}$ component, in the first scenario presented in Section 4.A.1. The dotted horizontal line represents the pan-chromatic cutoff frequency.

shows the cutoff frequency that would be achieved as a function of the relative estimation error of each $A_{c}$ component. The dotted horizontal line represents the pan-chromatic cutoff frequency. We can see that a relatively large uncertainty in any $A_{c}$ component would still ensure improvement over the panchromatic case, even though the performance is less than optimal. So, in this scenario, the cutoff frequency is insensitive to an error in $\left\{A_{c}\right\}$.

Even if no signal and noise STD estimation is available, the fusion weight vector $\boldsymbol{\alpha}$ can be sought empirically: at low SNR, a correct $\boldsymbol{\alpha}$ reveals details in what otherwise appears as random noise. 


\section{DISCUSSION}

We analyzed an effective limiting cutoff that can be achieved in a given color scenario, with given system properties, based on linear systems theory. Our framework can serve as a guideline when designing a polychromatic imaging system and a tool for performance evaluation. We presented a unified spatiospectral domain framework, resulting in limiting cutoff frequencies. Our work generalizes Ref. [2], which studies the limiting cutoff frequency in a single-channel, monochrome scenario. By utilizing a known linear technique for spectral fusion, we derived an analytical expression for the fused SNR. This increase in SNR expresses the objective benefit of color information, over a pan-chromatic system with the same total number of photons entering the system. Finally, we propose a modified, color-enhanced expression for the limiting cutoff frequency. We demonstrated our approach to assessing limiting cutoff frequency in color images, by numerical examples, simulations, and a demonstration using a real hyperspectral image.

Analysis should also be done for the Bayer CFA sensor, which is common in color imaging. Additional work can deal with alternative fusion operations and nonwhite random noise. Our analysis neglected a per-color phase component, following analysis performed on natural RGB images [33]. In future work, it is worth considering image domains other than RGB and hyperspectral visible data.

\section{APPENDIX A: DERIVATION OF SNR fused}

Let $\mathrm{SNR}_{\text {fused }}^{\text {out }}$ be the square root of the largest eigenvalue $\lambda$ of $\mathbf{C}_{\mathbf{n}}^{-1} \mathbf{C}_{\mathbf{A}}$. The matrix $\mathbf{C}_{\mathbf{n}}$ is diagonal with the per-channel noise variance, $\sigma_{c}^{2}$ as its eigenvalues. Thus, $\mathbf{C}_{\mathbf{n}}^{-1}$ takes the form

$$
\mathbf{C}_{\mathbf{n}}^{-1}=\left(\begin{array}{cccc}
\frac{1}{\sigma_{1}^{2}} & & \cdots & 0 \\
& \frac{1}{\sigma_{2}^{2}} & & \vdots \\
\vdots & & \ddots & \\
0 & \cdots & & \frac{1}{\sigma_{N}^{2}}
\end{array}\right)
$$

The Matrix $\mathbf{C}_{\mathbf{A}}$ is

$$
\mathbf{C}_{\mathbf{A}}=\left(\begin{array}{cccc}
A_{1}^{2} & A_{1} A_{2} & \cdots & A_{1} A_{N} \\
A_{2} A_{1} & A_{2}^{2} & & \vdots \\
\vdots & & \ddots & \\
A_{N} A_{1} & \cdots & & A_{N}^{2}
\end{array}\right)
$$

Thus, $\mathbf{C}_{\mathbf{n}}^{-1} \mathbf{C}_{\mathbf{A}}$ is

$$
\mathbf{C}_{\mathbf{n}}^{-1} \mathbf{C}_{\mathbf{A}}=\left(\begin{array}{cccc}
\frac{A_{1}^{2}}{\sigma_{1}^{2}} & \frac{A_{1} A_{2}}{\sigma_{1}^{2}} & \cdots & \frac{A_{1} A_{N}}{\sigma_{1}^{2}} \\
\frac{A_{2} A_{1}}{\sigma_{2}^{2}} & \frac{A_{2}^{2}}{\sigma_{2}^{2}} & & \vdots \\
\vdots & & \ddots & \\
\frac{A_{N} A_{1}}{\sigma_{N}^{2}} & \cdots & & \frac{A_{N}^{2}}{\sigma_{N}^{2}}
\end{array}\right)
$$

Let $\hat{\mathbf{l}}_{i}$ and $\hat{\mathbf{l}}_{j}$ be the $i$ th and $j$ th columns of $\mathbf{C}_{\mathbf{n}}^{-1} \mathbf{C}_{\mathbf{A}}$, respectively. It is evident that $\hat{\mathbf{l}}_{j}=\frac{A_{j}}{A_{i}} \hat{\mathbf{l}}_{i}, \forall i, j$. Thus, all columns of $\mathbf{C}_{\mathbf{n}}^{-1} \mathbf{C}_{\mathbf{A}}$ are linearly dependent, and $\operatorname{rank}\left(\mathbf{C}_{\mathbf{n}}^{-1} \mathbf{C}_{\mathbf{A}}\right)=1$. The number of nonzero eigenvalues of a matrix $\mathbf{M}$ is at most $\operatorname{rank}(\mathbf{M})$
[49]. Therefore, $\mathbf{C}_{\mathbf{n}}^{-1} \mathbf{C}_{\mathbf{A}}$ has one eigenvalue at most. Another theorem [50] states that trace(M) equals the sum of its eigenvalues. In our case, the sum of the eigenvalues of $\mathbf{C}_{n}^{-1} \mathbf{C}_{\mathbf{A}}$ equals to its only nonzero eigenvalue (if one such exists). Therefore,

$$
\mathrm{SNR}_{\text {fused }}^{\text {out }}=\sqrt{\lambda}=\sqrt{\operatorname{trace}\left(\mathbf{C}_{\mathbf{n}}^{-1} \mathbf{C}_{\mathbf{A}}\right)}=\sqrt{\sum_{c=1}^{N}\left[\mathrm{SNR}_{c}^{\mathrm{in}}\right]^{2}} .
$$

\section{ACKNOWLEDGMENTS}

The work was supported in part by the Israel Science Foundation (Grant No. 1467/12) and the VPR Fund of the Technion. This work was conducted in the Ollendorff Minerva Center. Minerva is funded through the BMBF.

\section{REFERENCES}

1. T. Treibitz and Y. Y. Schechner, "Recovery limits in pointwise degradation," in Proceedings of the IEEE International Conference on Computational Photography (2009).

2. T. Treibitz and Y. Y. Schechner, "Resolution loss without imaging blur," J. Opt. Soc. Am. A 29, 1516-1528 (2012).

3. H. Farid and E. H. Adelson, "Separating reflections from images by use of independent component analysis," J. Opt. Soc. Am. A 16, 2136-2145 (1999).

4. S. K. Nayar, X. S. Fang, and T. Boult, "Separation of reflection components using color and polarization," Int. J. Comput. Vis. 21, 163-186 (1997).

5. G. Petschnigg, R. Szeliski, M. Agrawala, M. Cohen, H. Hoppe, and K. Toyama, "Digital photography with flash and no-flash image pairs," ACM Trans. Graph. 23, 664-672 (2004).

6. R. Fattal, "Single image dehazing," ACM Trans. Graph. 27, 72 (2008).

7. K. He, J. Sun, and X. Tang, "Single image haze removal using dark channel prior," in IEEE Conference on Computer Vision and Pattern Recognition (2009), pp. 1956-1963.

8. N. Joshi and M. Cohen, "Seeing Mt. Rainier: lucky imaging for multi-image denoising, sharpening, and haze removal," in Proceedings of the IEEE International Conference on Computational Photography (2010), pp. 1-8.

9. J. Kopf, B. Neubert, B. Chen, M. Cohen, D. Cohen-Or, O. Deussen, M. Uyttendaele, and D. Lischinski, "Deep photo: model-based photograph enhancement and viewing," ACM Trans. Graph. 27, 116 (2008).

10. Y. Y. Schechner, S. G. Narasimhan, and S. K. Nayar, "Polarizationbased vision through haze," Appl. Opt. 42, 511-525 (2003).

11. R. T. Tan, "Visibility in bad weather from a single image," in IEEE Conference on Computer Vision and Pattern Recognition (2008).

12. J. Gu, R. Ramamoorthi, P. Belhumeur, and S. Nayar, "Dirty glass: rendering contamination on transparent surfaces," in Proceedings of the Eurographics Symposium on Rendering (2007), pp. $159-170$

13. Y. Haeghen, J. Naeyaert, I. Lemahieu, and W. Philips, “An imaging system with calibrated color image acquisition for use in dermatology," IEEE Trans. Med. Imaging 19, 722-730 (2000).

14. M. Robinson, E. Malaret, and T. White, "A radiometric calibration for the clementine hires camera," J. Geophys. Res. 180, 1-9 (2003).

15. E. Pinsky, A. Siman-Tov, and D. Peles, "Adaptive optimal spectral range for dynamically changing scene," Proc. SPIE 8407, $84070 \mathrm{~N}$ (2012).

16. A. Chakrabarti and T. Zickler, "Statistics of real-world hyperspectral images," in IEEE Conference on Computer Vision and Pattern Recognition (2011), pp. 193-200.

17. M. Kim, T. Ritschel, and J. Kautz, "Edge-aware color appearance," ACM Trans. Graph. 30, 13 (2011).

18. M. Kim, T. Weyrich, and J. Kautz, "Modeling human color perception under extended luminance levels," ACM Trans. Graph. 28, 27 (2009). 
19. K. Krapels, T. Jones, R. Driggers, and B. Teaney, "Target detection in color imagery: on the path to a color target acquisition model," Proc. SPIE 5612, 295-303 (2004).

20. R. Vollmerhausen, J. Olson, and J. Reynolds, "Modeling the benefit of color in target acquisition: characterizing color vision," Proc. SPIE 6941, 694105 (2008).

21. X. Cao, X. Tong, Q. Dai, and S. Lin, "High resolution multispectral video capture with a hybrid camera system," in IEEE Conference on Computer Vision and Pattern Recognition (2011), pp. 297-304.

22. R. Kawakami, J. Wright, Y. Tai, Y. Matsushita, M. Ben-Ezra, and K. Ikeuchi, "High-resolution hyperspectral imaging via matrix factorization," in IEEE Conference on Computer Vision and Pattern Recognition (2011), pp. 2329-2336.

23. A. Zomet and S. Peleg, "Multi-sensor super-resolution," in Proceedings of the IEEE Workshop on Applications of Computer Vision (2011), pp. 27-31.

24. K. Martinez, J. Cupitt, and D. R. Saunders, "High-resolution colorimetric imaging of paintings," Proc. SPIE 1901, 25-36 (1993).

25. F. H. Imai, M. R. Rosen, and R. S. Berns, "Comparison of spectrally narrow-band capture versus wide-band with a priori sample analysis for spectral reflectance estimation," in Proceedings of the Eighth Color Imaging Conference (2000), Vol. 234.

26. D. H. Foster, S. M. Nascimento, and K. Amano, "Information limits on neural identification of colored surfaces in natural scenes," Vis. Neurosci. 21, 331-336 (2004).

27. S. Hordley, G. Finalyson, and P. Morovic, "A multi-spectral image database and its application to image rendering across illumination," in IEEE Proceedings of the International Conference on Image and Graphics (2004), pp. 394-397.

28. J.-I. Park, M.-H. Lee, M. D. Grossberg, and S. K. Nayar, "Multispectral imaging using multiplexed illumination," in Proceedings of the IEEE Conference on Computer Vision (2007), pp. $1-8$.

29. M. Parmar, S. Lansel, and B. A. Wandell, "Spatio-spectral reconstruction of the multispectral datacube using sparse recovery," in IEEE International Conference on Image Processing (2008), pp. 473-476.

30. H. Du, X. Tong, X. Cao, and S. Lin, "A prism-based system for multispectral video acquisition," in Proceedings of the IEEE Conference on Computer Vision (2009), pp. 175-182.

31. F. Yasuma, T. Mitsunaga, D. Iso, and S. K. Nayar, "Generalized assorted pixel camera: postcapture control of resolution, dynamic range, and spectrum," IEEE Trans. Image Process. 19, 2241-2253 (2010).

32. B. E. Bayer, "Color imaging array," U.S. patent 3,971,065 (July 20, 1976)

33. A. Golts and Y. Y. Schechner, "Spatial frequency response of Bayer color image formation," Technion CCIT Technical Report 868, EE Pub. No. 1825 (Technion, 2014).
34. S. Bobrov and Y. Y. Schechner, "Image-based prediction of imaging and vision performance,” J. Opt. Soc. Am. A 24, 1920-1929 (2007).

35. N. Kopeika, A System Engineering Approach to Imaging (SPIE, 1998), Chaps. 9, 10, and 19.

36. L. Schaul, C. Fredembach, and S. Susstrunk, "Color image dehazing using the near-infrared," in IEEE International Conference on Image Processing (2009), pp. 1629-1632.

37. O. Cossairt, M. Gupta, and S. Nayar, "When does computational imaging improve performance?" Columbia University Computer Science Technical Report CUCS-004-12 (2012).

38. S. Hasinoff, F. Durand, and W. Freeman, "Noise-optimal capture for high dynamic range photography," in IEEE Conference on Computer Vision and Pattern Recognition (2010), pp. 553-560.

39. J. Takamatsu, Y. Matsushita, and K. Ikeuchi, "Estimating radiometric response functions from image noise variance," in Proceedings of the European Conference on Computer Vision (Springer, 2008), pp. 623-637.

40. C. Liu, R. Szeliski, S. Kang, C. Zitnick, and W. Freeman, "Automatic estimation and removal of noise from a single image," IEEE Trans. Pattern Anal. Mach. Intell. 30, 299-314 (2008).

41. Y. Matsushita and S. Lin, "Radiometric calibration from noise distributions," in IEEE Conference on Computer Vision and Pattern Recognition (2007).

42. G. Healey and R. Kondepudy, "Radiometric CCD camera calibration and noise estimation," IEEE Trans. Pattern Anal. Mach Intell. 16, 267-276 (1994).

43. A. Wenger, A. Gardner, C. Tchou, J. Unger, T. Hawkins, and P. Debevec, "Performance relighting and reflectance transformation with time-multiplexed illumination," ACM Trans. Graph. 24, 756-764 (2005)

44. Y. Y. Schechner, S. K. Nayar, and P. N. Belhumeur, "Multiplexing for optimal lighting," IEEE Trans. Pattern Anal. Mach. Intell. 29, 1339-1354 (2007).

45. R. F. Lyon and P. M. Hubel, "Eyeing the camera: into the next century," in Color and Imaging Conference (Society for Imaging Science and Technology, 2002), Vol. 2002, pp. 349-355.

46. R. B. Merrill, "Color separation in an active pixel cell imaging array using a triple-well structure," U.S. patent 5,965,875 (October 12, 1999).

47. C. C. S. Tianpeng Wang and C.-C. Li, "Pixelwise fusion for optimizing SNR in multiple-plate computed radiography imaging," IEEE Trans. Med. Imaging 18, 239-251 (1999).

48. K. Andersen, "An elementary proof of Rayleigh's principle," Int. J. Math. Edu. Sci. Technol. 31, 449-453, 2000.

49. C. D. Manning, P. Raghavan, and H. Schtze, Introduction to Information Retrieval, Online Edition (Cambridge University, 2009).

50. E. Dummit, Linear Algebra (320) Course Handouts, Part 3: Eigenvalues and Eigenvectors, http://www.math.wisc.edu/ $\sim$ dummit/handouts.html. 